\title{
ARTICLE
}

Clinical Study

\section{Tamoxifen treatment for male breast cancer and risk of thromboembolism: prospective cohort analysis}

\author{
Holm Eggemann ${ }^{1}$, Anna-Lena Bernreiter ${ }^{1}$, Mattea Reinisch ${ }^{2}$, Sibylle Loibl ${ }^{3}$, Florin-Andrei Taran ${ }^{4}$, Serban-Dan Costa ${ }^{1}$ and \\ Atanas Ignatov ${ }^{1,5}$
}

PURPOSE: Thromboembolism is a common adverse event in women treated with tamoxifen (TAM) for breast cancer. The risk in male breast cancer patients is poorly investigated. We aimed to examine the risk of thrombotic events after TAM in male breast cancer patients.

PATIENTS AND METHODS: In this prospective cohort study, 448 patients treated between May 2009 and July 2017 for male breast cancer (BC) were assessed for eligibility. Patients with follow-up shorter than 6 months were excluded. The cumulative risk of thromboembolism was evaluated.

RESULTS: The median follow-up was 47 months (range 6-101 months) with a median age of 69.4 years (range 27-89 years). Oestrogen receptor and progesterone receptor expression levels were observed in 98.3 and $94.9 \%$ of cases, respectively. During the follow-up period, thrombotic events were documented in 21 (11.9\%) of 177 patients receiving TAM and in 1 (2.5\%) of 41 patients who did not receive tamoxifen. The estimated incidence was 51.9 per 1000 person-years and 21.5 per 1000 person-years, respectively. Notably, the highest risk was identified in the first 18 months, where $81 \%$ of the observed thrombotic events occurred. Patients aged older than 71 years had a significantly increased risk of thrombotic event under TAM treatment than their younger counterparts $(p=0.033)$. History of thrombotic event, cardiovascular and liver disease, as well as additional adjuvant treatment were not associated with increased thrombotic risk.

CONCLUSION: The risk of thrombotic event in men treated with TAM for breast cancer is markedly increased in the first 18 months of treatment, and should be considered during treatment decisions.

British Journal of Cancer (2019) 120:301-305; https://doi.org/10.1038/s41416-018-0369-2

\section{INTRODUCTION}

Male breast cancer (BC) is an uncommon disease and its rarity makes the performance of prospective randomised trials very difficult. As a result, the treatment concepts are based on limited retrospective studies and clinical management of the female $B C{ }^{1}$ Male BC appears to be hormone receptor (HR)-positive in most cases and endocrine therapy is the most important treatment option. In a recent retrospective study of 257 male BC patients, we showed that adjuvant treatment with TAM was associated with a 1.4-fold decreased risk of cancer mortality compared to $\mathrm{Al}$ treatment. ${ }^{2}$ Via matching analysis among male and female patients with hormone receptor-positive breast cancer, we demonstrated clearly that the benefit of TAM treatment in male $B C$ is comparable with the effect of TAM in female $B C^{3}$

One of the most common TAM-associated adverse effects is thromboembolic events. ${ }^{4,5}$ In general, women with $B C$ have an increased risk of thrombotic events compared to women without $\mathrm{BC}^{6}$ However, thus far the adverse effects of TAM in male BC have been poorly investigated. Therefore, identifying the risk profile of TAM in men will help us to further improve the treatment of male BC.
In this large prospective cohort study, the risk of deep-venous thrombosis and thromboembolism in men treated with TAM for $\mathrm{BC}$ was investigated. The impact of other risk factors on thrombotic events were also examined.

\section{MATERIALS AND METHODS}

We investigated cases of male $B C$ from the national prospective cancer registry of Germany. This tumour register contains information about male BC patients: date of diagnosis, patients and tumour characteristics, operative and neo- and/or adjuvanttreatment, date and localisation of relapse, date and cause of death, secondary cancer, comorbidities. We analysed 448 men with primary BC diagnosed between May 2009 and July 2017. We included only patients with non-metastatic invasive HR-positive BC and who had a minimal follow-up of 6 months. Patients were excluded if the endocrine treatment was not defined $(n=124)$ or the follow-up was within 6 months $(n=106)$. Accordingly, of these 448 patients, 218 were eligible for analysis (Fig. 1). The trial was undertaken in accordance with the Declaration of Helsinki and guidelines for Good Clinical Practice and was approved by the

\footnotetext{
${ }^{1}$ Department of Obstetrics and Gynaecology, Otto-von-Guericke University, Magdeburg, Germany; ${ }^{2}$ Breast Unit, Kliniken Essen-Mitte, Essen, Germany; ${ }^{3}$ GBG Forschungs GmbH, Neu-Isenburg, Germany; ${ }^{4}$ Department of Obstetrics and Gynaecology, University Hospital Tübingen, Tübingen, Germany and ${ }^{5}$ Department of Gynaecology and Obstetrics, University Medical Center, Regensburg, Germany

Correspondence: Holm Eggemann (holm.eggemann@med.ovgu.de) or Atanas Ignatov (atanas.ignatov@gmail.com)
}

Received: 11 September 2018 Revised: 2 December 2018 Accepted: 5 December 2018

Published online: 17 January 2019 


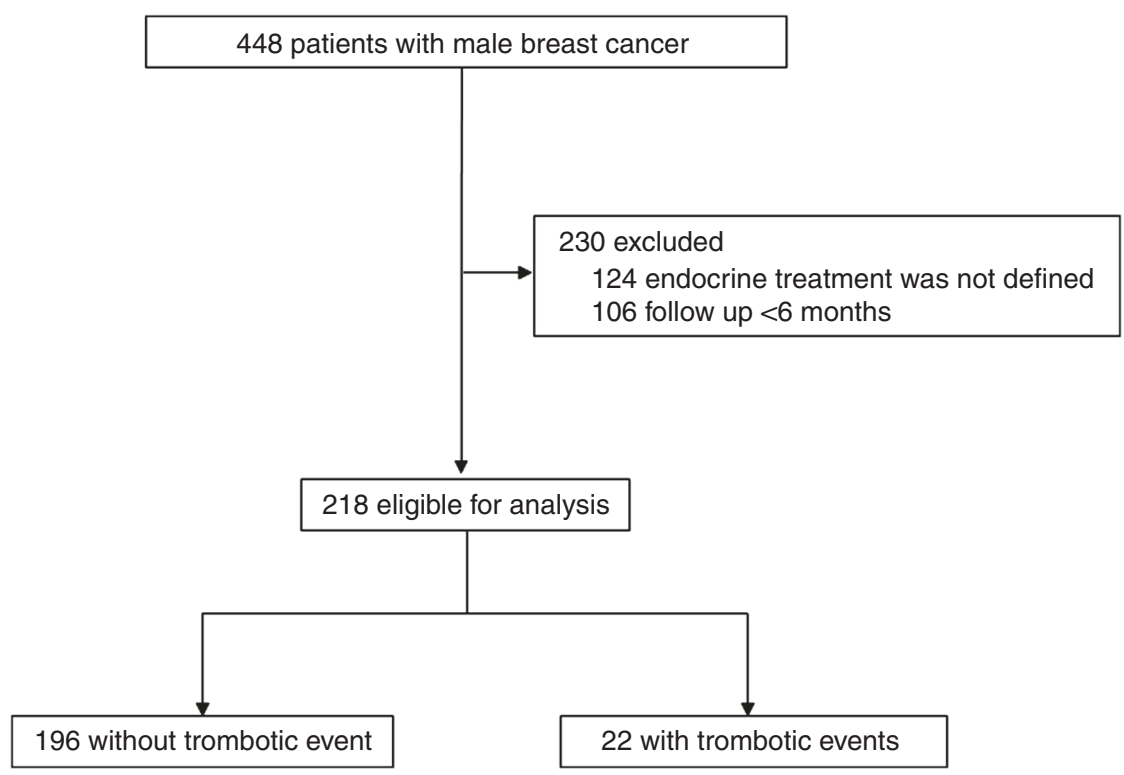

Fig. 1 Study design

Research and Ethical Committee of Otto-von-Guericke University, Magdeburg, Germany. Patients gave written informed consent for data-transfer to the tumour registry before treatment. This trial is registered at the international clinical trial registry platform under the number DRKS00009536 (https://drks-neu.uniklinik-freiburg.de/ drks_web/navigate.do?navigationld=trial.

HTML\&TRIAL_ID=DRKS00009536).

The primary outcome of the study was the rate of thrombotic events under TAM treatment. Thrombotic events were defined as deep-venous thrombosis and/or pulmonary embolism. As a secondary outcome we investigated the influence of other risk factors on thrombotic events in TAM-treated cohort of patients. From each patient the following information was collected: age, year of diagnosis, height and weight, comorbidity, history of thrombotic event, tumour characteristics, tumour treatment strategy, start and stop of TAM treatment, and reason for discontinuation of TAM.

Statistical analysis

The statistical analyses were performed using SPSS Version 22.0 (SPSS, Chicago, IL, USA). Associations between tumour, patient and treatment characteristics with thrombotic events were analysed by cross-tabulation and tested using the $X^{2}$ test or Fisher's exact test. Survival probability was studied using the Kaplan-Meier method. The equality of survival curves was tested using the log-rank test. Cox proportional hazards models were used to assess the influence of adjuvant treatment as an independent prognostic factor and to control further for confounding bias. All tests were two-sided and determined statistically significant if the $p$-value was $\leq 0.05$.

\section{RESULTS}

Between May 2009 and July 2017, 448 men with primary nonmetastatic BC were identified and 230 were excluded (Fig. 1). The median follow-up was 47 months (range 6-101 months) and the median age at diagnosis was 69.4 years (range $27-89$ years).

In this cohort of patients with male BC, positive estrogenic and progesterone receptor status was observed in 97.7 and $94.0 \%$, respectively. HER2 positivity was demonstrated for $18.1 \%$ of the tumours (Table 1). Lymph nodes were involved in $44.1 \%$, lymph vessels in $40.6 \%$ and blood vessels in $5.3 \%$. Most of the patients $(99.1 \%)$ received surgery, 52.4 and $42.6 \%$ received radiotherapy and chemotherapy, respectively. Most of the tumours were of intermediate grade of differentiation (62.0\%) and had a tumour diameter of $<20 \mathrm{~mm}(86.3 \%)$.

After further exclusion, 218 men with HR-positive BC were eligible for analysis. Among them, 177 were treated with TAM and 41 received no TAM. In the group of TAM-treated patients 21 (11.9\%) experienced a thrombotic event as a side effect. The estimated incidence of thrombotic events in tamoxifen-treated patients was 51.9 of 1000 person-years. Next, we investigated the relationship between the duration of TAM treatment and incidence of thrombotic events. The highest incidence of $6.2 \%$ (21.6 per 1000 person-years) was observed in the first year of TAM treatment. In the 2nd and in the 3rdyear 3.4 (13.6 per 1000 person-years) and 2.3\% (11.4 per 1000 person-years) of thromboembolism events were observed, respectively. The event-free survival is shown in Fig. 2. During the next 2 years, no additional cases were found. The cumulative incidence curve suggested no further increased risk after the first 3 years of TAM therapy. Thus, the occurrence of thrombosis and embolism had a sharper rise in the first 18 months with a cumulative incidence of $81 \%$ of all thrombotic events. In the group of patients who did not received tamoxifen only, 1 (2.5\%) thrombotic event was observed in all 41 cases with an incidence of 21.5 of 1000 person-years.

Furthermore, we investigated the influence of other tumour patients and their respective treatment characteristics on thrombotic events. As demonstrated in Table 2, there were no clinical and pathological factors associated with an increased risk of thrombosis in this group of TAM-treated patients. The discontinuation rate of TAM treatment was also examined. TAM was discontinued in $40(22.6 \%)$ of 177 patients (data not shown). Interestingly, in $17(42.5 \%)$ of 40 cases TAM discontinuation was caused by tumour progression and/or relapse. In the remaining 23 (56.5\%) patients TAM-induced side effects were the reason for discontinuation.

\section{DISCUSSION}

We determined the rate of TAM-induced thrombotic events in patients with male $\mathrm{BC}$, and found that the cumulative risk of thrombosis and embolism is $11.9 \%$ and more than $80 \%$ of these thrombotic events occurred in the first 18 months after initiation of treatment. The incidence of thrombotic events in tamoxifentreated patients was 51.9 per 1000 person-years. In the group of 
Table 1. Clinical and pathological characteristics

\begin{tabular}{|c|c|c|}
\hline Parameter & $N$ & $\%$ \\
\hline Median age (years) & $70.1(27-90)$ & \\
\hline $\mathrm{BMI}, \mathrm{kg} / \mathrm{m}^{2}$ & $27.4(17-45)$ & \\
\hline \multicolumn{3}{|l|}{ Tumor (T) stage } \\
\hline 0 & 7 & \\
\hline 1 & 89 & 3.3 \\
\hline 2 & 71 & 42.0 \\
\hline 3 & 6 & 41.0 \\
\hline 4 & 23 & 2.8 \\
\hline Missing & 6 & 10.8 \\
\hline \multicolumn{3}{|l|}{ Lymph node status } \\
\hline Negative & 113 & \\
\hline Positive & 89 & 55.9 \\
\hline Missing & 16 & 44.1 \\
\hline \multicolumn{3}{|l|}{ Histological grade } \\
\hline 1 & 21 & \\
\hline 2 & 132 & 9.9 \\
\hline 3 & 60 & 62.0 \\
\hline Missing & 5 & 28.2 \\
\hline \multicolumn{3}{|c|}{ Blood vessel involvement } \\
\hline Negative & 178 & \\
\hline Positive & 10 & 94.7 \\
\hline Missing & 30 & 5.3 \\
\hline \multicolumn{3}{|c|}{ Lymph vessel involvement } \\
\hline Negative & 114 & \\
\hline Positive & 78 & 59.4 \\
\hline Missing & 26 & 40.6 \\
\hline \multicolumn{3}{|l|}{ ER status } \\
\hline Negative & 5 & \\
\hline Positive & 210 & 2.3 \\
\hline Missing & 3 & 97.7 \\
\hline \multicolumn{3}{|l|}{$P R$ status } \\
\hline Negative & 13 & \\
\hline Positive & 202 & 6.0 \\
\hline Missing & 3 & 94.0 \\
\hline \multicolumn{3}{|l|}{ HER2 status } \\
\hline Negative & 145 & \\
\hline Positive & 32 & 81.9 \\
\hline Missing & 41 & 18.1 \\
\hline \multicolumn{3}{|l|}{ Operative therapy } \\
\hline No & 2 & \\
\hline Yes & 209 & 0.9 \\
\hline Missing & 7 & 99.1 \\
\hline \multicolumn{3}{|l|}{ Radiotherapy } \\
\hline No & 100 & \\
\hline Yes & 110 & 47.6 \\
\hline Missing & 8 & 52.4 \\
\hline \multicolumn{3}{|l|}{ Chemotherapy } \\
\hline No & 124 & \\
\hline Yes & 92 & 57.4 \\
\hline Missing & 2 & 42.6 \\
\hline
\end{tabular}

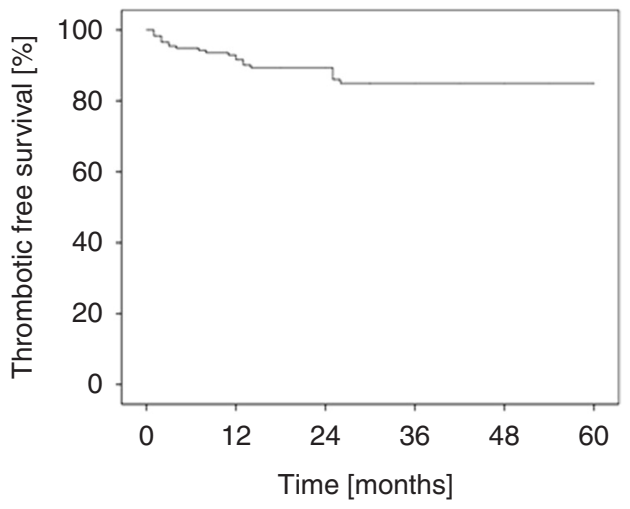

Follow-up Nr. $\begin{array}{llllll}177 & 131 & 82 & 61 & 45 & 23\end{array}$

Fig. 2 Cumulative incidence of thrombotic events in the first five years of TAM treatment

Table 2. Adjusted risk of thromboebolism

\begin{tabular}{|c|c|c|}
\hline Variable & Hazard ratio for events $(95 \% \mathrm{Cl})$ & $P$ value \\
\hline \multicolumn{3}{|c|}{ Age at diagnosis (years) } \\
\hline$\leq 70$ & 1.00 & 0.221 \\
\hline$>70$ & $2.32(0.60-8.98)$ & \\
\hline \multicolumn{3}{|c|}{ Tumor $(T)$ size } \\
\hline$\leq 20 \mathrm{~mm}$ & 1.00 & 0.726 \\
\hline$>20 \mathrm{~mm}$ & $0.68(0.80-5.82)$ & \\
\hline \multicolumn{3}{|c|}{ Histological grade } \\
\hline 1,2 & 1.00 & 0.650 \\
\hline 3 & $1.28(0.32-5.09)$ & \\
\hline \multicolumn{3}{|c|}{ Lymph node status } \\
\hline Negativ & 1.00 & 0.650 \\
\hline Positiv & $0.70(0.15-3.24)$ & \\
\hline \multicolumn{3}{|c|}{ HER2 status } \\
\hline Negative & 1.00 & 0.782 \\
\hline Positive & $0.73(0.08-6.59)$ & \\
\hline \multicolumn{3}{|c|}{ Blood vessel involvement } \\
\hline Negative & 1.00 & 0.491 \\
\hline Positive & $2.20(0.23-20.88)$ & \\
\hline \multicolumn{3}{|c|}{ Lymph vessel involvement } \\
\hline Negative & 1.00 & 0.569 \\
\hline Positive & $1.54(0.35-6.72)$ & \\
\hline \multicolumn{3}{|c|}{ Chemotherapy } \\
\hline No & 1.00 & 0.375 \\
\hline Yes & $0.52(0.12-2.23)$ & \\
\hline \multicolumn{3}{|l|}{ Radiation } \\
\hline No & 1.00 & 0.258 \\
\hline Yes & $0.46(0.12-1.77)$ & \\
\hline
\end{tabular}

patients treated without tamoxifen the thrombotic events were observed in 21.5 per 1000 person-years.

In accordance with previous trials with female $\mathrm{BC}_{1}^{4,7,8}$ we have found that TAM is associated with a markedly increased risk of thrombosis. Interestingly, the estimated rate of thrombotic events in our cohort with male BC is somehow higher than the observed 
rates of thrombosis in women. However, compliant to our data, a retrospective study with male BC thrombotic events were recorded in 6 (9\%) out of 64 patients. ${ }^{9}$ In another study with 24 male BC patients, TAM-induced deep-venous thrombosis was observed in $4.2 \% .{ }^{10}$ The lower rate of events in the study could be explain by the small number of events $(n=1)$ or by the fact that only deep-venous thrombosis was evaluated, and no information exists about the rate of venous embolism. All this data suggests a slightly elevated risk of thrombosis in men with $\mathrm{BC}$ compared to women with BC. Gender-specific risk of thrombotic events has been investigated in numerous trials. Some of them have shown that women exhibit a lower risk of recurrent venous thromboembolism than men, but this data could not be confirmed in other studies. ${ }^{11}$ The reason for this discrepancy is unclear and should be further investigated.

Along with the gender discrepancy, this study reveals another important finding. The incidence of thromboembolism was very high in the first 1.5 years and did not increase after the 3rd year of TAM treatment. Although, this observation is a known phenomenon in women with $B C,{ }^{6,7,12,13}$ our report is the first demonstrating such a steep increase of thrombotic events after initiation of TAM treatment for male BC. In a large population-based study women treated with TAM were at a higher risk for thromboembolism during the first 2 years after initiation of TAM treatment. ${ }^{7}$ Onitilo et al. and Walker et al. have also suggested that TAMinduced thrombotic events occurred early during TAM treatment. ${ }^{6,12}$ The reason for this phenomenon is not well-understood. One explanation is the agonistic activity of TAM on oestrogen receptor ${ }^{14,15}$ and is supported by the finding that hormone replacement therapy on female $B C$ patients is characterised with a similar elevated risk of thrombosis during the initial years of treatment. ${ }^{16}$ Supporting this, an elevated risk of thrombosis has been observed with other antihormonal treatments in patients with prostate cancer. ${ }^{17}$ In general, the effect of hormone therapy on coagulation is multifactorial and includes increased levels/ activity of pro-coagulation factors and inhibition of coagulation inhibitors. $^{18}$ An adaptation of the coagulation system to the procoagulant effects of TAM after 1-2 years is proposed. Furthermore, the occurrence of most thrombotic events in the early period of TAM treatment is more likely caused by the influence of conventional risk factors including older age, prolonged immobilisation, extensive surgery, history of stroke and heart disease etc., ${ }^{43}$ Notably, in our cohort of patients with male BC only older age was associated with increased risk of thrombotic events. Men older than 70 years were at higher risk of thrombosis than their younger counterparts. However, various other risk factors could not be linked to a further increase of TAMinduced thromboembolism.

This observation is very important with regards to managing the risk of thrombosis. Monitoring using clinical examination and different algorithms should be performed during the period of highest risk. ${ }^{19}$ If possible, TAM should be discontinued if additional risk factors for thromboembolism are present, such as major surgery, prolonged immobilisation, stroke or trauma. ${ }^{13}$ Data from studies comparing female $B C$ and male $B C$ suggest that male $B C$ is largely HR-positive, and the rates of oestrogen and progesterone receptors vary between 70 and $90 \%$ indicating that TAM is the most important systemic therapy in male BC. ${ }^{1,20}$ We observed an even higher rate of hormone receptor positivity. Oestrogen and progesterone receptors were positive in 98.3 and $94.9 \%$ of the cases, respectively. As we already reported treatment with aromatase inhibitors is inferior to TAM treatment for male BC and the effect of TAM in male $B C$ is comparable with that in female $\mathrm{BC}^{2,3}$ In this context, TAM treatment remains the treatment of choice for HR-positive male BC. In the present study, discontinuation of TAM was observed in $22.6 \%$ and is in complete agreement with others. ${ }^{9,10}$ To note, the rate of discontinuation due to TAM-induced side effects was $13 \%$. The remaining discontinuations were due to tumour progression or relapse.

The most important limitation of the present study is the lack of a control group untreated with TAM. It is caused by the small number of patients who did not receive TAM. Thus, we were unable to compare the hazard ratio of TAM with the untreated population and evaluate the influence of additional risk factors on thrombotic risk. Furthermore, we did not evaluate the influence of inherited risk factors on the appearance of thrombotic events in more detail.

However, our study had several important strengths. First, the study is prospective, with a corresponding well-maintained documentation. Second, this is the largest study of adjuvant TAM therapy in male BC patients. Third, this study is populationbased, and the exclusion criteria are kept to a minimum resulting in a high level of external validity.

In conclusion, these results will support physicians in treatment decision of patients with male $B C$ and will sensitise them to focus their attention during the first 1-2 years of treatment. In this period an appropriate monitoring of the patients is warranted.

\section{ADDITIONAL INFORMATION}

Competing interests: The authors declare no conflict of interest.

Ethical approval: All the research meets the ethical guidelines, including adherence to the legal requirements of the study country. In accordance with the statement of the Research and Ethical Committee of the Otto-von-Guericke University, Magdeburg, Germany, written informed consent was obtained from each participant or participant's guardian before inclusion in the study. Huang $C$, Harada $Y$, Hosomi A, Masahara-Negishi $Y$, Seino J, Fujihira et al. Endo-beta-N-acetylglucosaminidase forms $\mathrm{N}$-GIcNAc protein aggregates during ER-associated degradation in Ngly1-defective cells. Proc. Natl. Acad. Sci. USA 2015; 112, 1398-1403.

Funding: This research did not receive any specific grant from any funding agency in the public, commercial or not-for-profit sector.

Note: This work is published under the standard license to publish agreement. After 12 months the work will become freely available and the license terms will switch to a Creative Commons Attribution 4.0 International (CC BY 4.0).

Publisher's note: Springer Nature remains neutral with regard to jurisdictional claims in published maps and institutional affiliations.

\section{REFERENCES}

1. Losurdo, A. et al. Controversies in clinicopathological characteristics and treatment strategies of male breast cancer: a review of the literature. Crit. Rev. Oncol. Hematol. 113, 283-291 (2017).

2. Eggemann, $\mathrm{H}$. et al. Adjuvant therapy with tamoxifen compared to aromatase inhibitors for 257 male breast cancer patients. Breast Cancer Res. Treat. 137, 465-470 (2013).

3. Eggemann, H., Altmann, U., Costa, S. D. \& Ignatov, A. Survival benefit of tamoxifen and aromatase inhibitor in male and female breast cancer. J. Cancer Res. Clin. Oncol. 144, 337-341 (2017).

4. Cuzick, J. et al. First results from the International Breast Cancer Intervention Study (IBIS-I): a randomised prevention trial. Lancet 360, 817-824 (2002).

5. Grady, D. et al. Postmenopausal hormone therapy increases risk for venous thromboembolic disease. The Heart and Estrogen/progestin Replacement Study. Ann. Intern. Med. 132, 689-696 (2000).

6. Walker, A. J., Card, T. R., West, J., Crooks, C. \& Grainge, M. J. Incidence of venous thromboembolism in patients with cancer-a cohort study using linked United Kingdom databases. Eur. J. Cancer 49, 1404-1413 (2013).

7. Hernandez, R. K., Sorensen, H. T., Pedersen, L., Jacobsen, J. \& Lash, T. L. Tamoxifen treatment and risk of deep venous thrombosis and pulmonary embolism: a Danish population-based cohort study. Cancer 115, 4442-4449 (2009).

8. Howell, A. et al. Results of the ATAC (arimidex, tamoxifen, alone or in combination) trial after completion of 5 years' adjuvant treatment for breast cancer. Lancet 365, 60-62 (2005). 
9. Pemmaraju, N., Munsell, M. F., Hortobagyi, G. N. \& Giordano, S. H. Retrospective review of male breast cancer patients: analysis of tamoxifen-related side-effects. Ann. Oncol. 23, 1471-1474 (2012).

10. Anelli, T. F., Anelli, A., Tran, K. N., Lebwohl, D. E. \& Borgen, P. I. Tamoxifen administration is associated with a high rate of treatment-limiting symptoms in male breast cancer patients. Cancer 74, 74-77 (1994).

11. Tormene, D., Ferri, V., Carraro, S. \& Simioni, P. Gender and the risk of venous thromboembolism. Semin. Thromb. Hemost. 37, 193-198 (2011).

12. Onitilo, A. A. et al. Clustering of venous thrombosis events at the start of tamoxifen therapy in breast cancer: a population-based experience. Thromb. Res. 130, 27-31 (2012).

13. Duggan, C., Marriott, K., Edwards, R. \& Cuzick, J. Inherited and acquired risk factors for venous thromboembolic disease among women taking tamoxifen to prevent breast cancer. J. Clin. Oncol. 21, 3588-3593 (2003).

14. Ignatov, A., Ignatov, T., Roessner, A., Costa, S. D. \& Kalinski, T. Role of GPR30 in the mechanisms of tamoxifen resistance in breast cancer MCF-7 cells. Breast Cancer Res. Treat. 123, 87-96 (2010).
15. Ignatov, A. et al. G-protein-coupled estrogen receptor GPR30 and tamoxifen resistance in breast cancer. Breast Cancer Res. Treat. 128, 457-466 (2011)

16. Curb, J. D. et al. Venous thrombosis and conjugated equine estrogen in women without a uterus. Arch. Intern. Med. 166, 772-780 (2006).

17. Smith, M. R. et al. Toremifene to reduce fracture risk in men receiving androgen deprivation therapy for prostate cancer. J. Urol. 184, 1316-1321 (2010).

18. Sandset, P. M., Hoibraaten, E., Eilertsen, A. L. \& Dahm, A. Mechanisms of thrombosis related to hormone therapy. Thromb. Res. 123(Suppl 2), S70-S73 (2009).

19. Gupta, R. T., Kakarla, R. K., Kirshenbaum, K. J. \& Tapson, V. F. D-dimers and efficacy of clinical risk estimation algorithms: sensitivity in evaluation of acute pulmonary embolism. Am. J. Roentgenol. 193, 425-430 (2009).

20. Anderson, W. F., Althuis, M. D., Brinton, L. A. \& Devesa, S. S. Is male breast cancer similar or different than female breast cancer? Breast Cancer Res. Treat. 83, 77-86 (2004). 